\title{
ON FINITE-DIMENSIONAL TORSION-FREE MODULES AND RINGS
}

\section{E. P. ARMENDARIZ}

1. This note is concerned primarily with a study of modules having zero singular submodule, called torsion-free modules, over finitedimensional rings. In some of our results we do not require that the ring be finite-dimensional but only that direct sums of torsion-free injective modules be injective, a property shown by Mark Teply to be equivalent to the ring containing no infinite direct sum of torsionfree left ideals. As might be expected, torsion-free modules over these latter rings behave very much like the usual torsion-free modules over (commutative) integral domains. For example, we show in Theorem 1 that direct sums of torsion-free injectives are injective if and only if every torsion-free module contains a unique maximal injective submodule. Also, in analogy to the case for Abelian groups, we establish that a torsion-free module over a finite-dimensional torsion-free ring contains a homomorphic image of every nonzero torsion-free module if and only if it contains a faithful injective submodule (Theorem 3). This enables us to show in Theorem 4 that a semiprime finite-dimensional torsion-free ring has a projective injective envelope if and only if it is semisimple Artinian. A result for torsion-free prime rings without any restricted chain conditions similar to Theorem 3 is also obtained. Finally, over self-injective finitedimensional rings we show that all torsion-free modules are injective and completely reducible (Corollary 6 ).

We assume throughout that $R$ is a ring with identity and all $R$-modules are unitary left $R$-modules.

2. The singular submodule of an $R$-module $M$ will be denoted by $Z(M)$. Following A. W. Goldie [6], $M$ is torsion-free if $Z(M)=0$ and $R$ is a torsion-free ring if $R$ is a torsion-free $R$-module. An $R$-module $M$ is finite-dimensional if it contains no infinite direct sum of submodules. For an $R$-module $M, E(M)$ will denote the injective envelope of $M$.

The following basic lemma is our starting point.

Lemma 1. Let $Q$ be an injective $R$-module and $M$ a torsion-free $R$ module. Then for any $\alpha \in \operatorname{Hom}_{R}(Q, M), \operatorname{Ker} \alpha$ is a direct summand of $Q$ and hence Im $\alpha$ is an injective $R$-module.

Received by the editors February 24, 1969. 
Proof. If $\alpha \in \operatorname{Hom}_{R}(Q, M)$ then Im $\alpha$ is torsion-free since $M$ is torsion-free. If $\operatorname{Ker} \alpha$ is an essential submodule of a submodule $P \subseteq Q$, then $Z(P / \operatorname{Ker} \alpha)=P / \operatorname{Ker} \alpha$ and it follows that $P / \operatorname{Ker} \alpha=0$, being isomorphic to a torsion-free module. Thus $\operatorname{Ker} \alpha$ has no essential extension in $Q$, hence $\operatorname{Ker} \alpha$ is injective and so a direct summand of $Q$.

The next lemma is well known and easily established.

Lemma 2. $A$ direct sum or direct product of torsion-free $R$-modules is torsion-free.

If a ring $R$ is left Noetherian then any direct sum of injective $R$-modules is injective [3, p. 17]. In [9, Theorem 2.1] Mark Teply has given several conditions on a ring $R$ which are equivalent to the following property (see also [8]):

$\left({ }^{*}\right)$ Any direct sum of torsion-free injective $R$-modules is injective. One of these, which we will use later, is that $R$ contain no infinite direct sum of torsion-free left ideals. Thus any finite-dimensional ring $R$ has property $\left({ }^{*}\right)$. Our first result gives another condition equivalent to $\left(^{*}\right)$ in terms of the injective submodules of torsion-free $R$-modules.

THEOREM 1. A necessary and sufficient condition for a ring $R$ to have property $\left({ }^{*}\right)$ is that every torsion-free $R$-module has a (unique) maximal injective submodule.

Proof. Suppose first that $R$ has property $\left({ }^{*}\right)$. Let $M$ be a torsionfree $R$-module, $\left\{H_{\lambda} \mid \lambda \in \Lambda\right\}$ the set of injective submodules of $M$, and $D=\sum_{\lambda \in \Lambda} H_{\lambda}$. If $H=\oplus \sum_{\lambda \in \Lambda} H_{\lambda}$, then by $\left(^{*}\right) H$ is injective since each $H_{\lambda}$ is torsion-free, and $D$ is a homomorphic image of $H$. Since $D$ is torsion-free, $D$ is injective by Lemma 1 . Evidently, $D$ is the maximal injective submodule of $M$. For the sufficiency let $Q$ $=\oplus \sum_{\lambda \in \Lambda} Q_{\lambda}$, where each $Q_{\lambda}$ is torsion-free and injective. Then $Q$ is torsion-free by Lemma 2 , so $Q$ has a maximal injective submodule $D \neq 0$. If $A$ is any injective submodule of $Q$, then $D+A$ is a torsionfree image of the injective module $D \oplus A$ hence is injective by Lemma 1. By maximality of $D, A \subseteq D$. In particular $Q_{\lambda} \subseteq D$ for all $\lambda \in \Lambda$ and thus $Q=D$ is injective.

We now give an application of Theorem 1 . We note first that since a torsion-free self-injective ring is regular, it is semisimple Artinian if and only if it is finite-dimensional.

THEOREM 2. If $R$ is a semiprime finite-dimensional torsion-free ring then $R=K \oplus S$ (ring direct sum), where $K$ is a semisimple Artinian ring and $S$ is a semiprime finite-dimensional torsion-free ring containing no nonzero S-injective left ideals. 
Proof. By Theorem 1, $R$ contains a maximal injective left ideal $K$, hence $R=K \oplus S$. If $r \in R$ then $K r$ is a homomorphic image of $K$ under right multiplication by $r$ and so $K r$ is an injective $R$-module by Lemma 1. Thus $K r \subseteq K$ and so $K$ is a two-sided ideal of $R$. Now $K S \subseteq K \cap S=0$ and so $(S K)^{2}=0$. Since $R$ contains no nonzero nilpotent ideals, $S K=0$ and this implies $S$ is also a two-sided ideal of $R$. The remaining assertions concerning $S$ are easily established.

If $R$ is a left Noetherian ring then $Z(R)$ is a nilpotent ideal of $R$, [5], so if in addition $R$ is semiprime then $Z(R)=0$. Thus we have

COROLlary 1. If $R$ is a left Noetherian semiprime ring then $R$ $=K \oplus S$ (ring direct sum), where $K$ is semisimple Artinian and $S$ is a left Noetherian semiprime ring containing no nonzero S-injective left ideals.

Corollary 2. A finite-dimensional ring $R$ is semisimple Artinian if and only if every nonzero left ideal contains a nonzero injective left ideal.

Proof. Since $Z(R)$ can contain no nonzero idempotents we have $Z(R)=0$. The same holds for any nilpotent left ideal. Thus $R$ is also semiprime.

Corollary 3. A finite-dimensional torsion-free prime ring $R$ is simple Artinian if and only if $R$ contains a nonzero injective left $i d e a l$.

If $R$ is a torsion-free ring then for an $R$-module $M, Z(M)=M$ if and only if $\operatorname{Hom}_{R}(M, E(R))=0$ [4, Proposition 1]. Thus the torsionfree $R$-modules are precisely those $R$-modules which are embeddable in a direct product of copies of $E(R)$. Thus $E(R)$ can be considered a cogenerator for the class of torsion-free $R$-modules. We will use Theorem 1 to characterize all torsion-free cogenerators for the torsion-free $R$-modules whenever $R$ is a finite-dimensional torsion-free ring. Note that a torsion-free $R$-module $M \neq 0$ is a cogenerator for the torsionfree $R$-modules if and only if $\operatorname{Hom}_{R}(B, M) \neq 0$ for all torsion-free $R$-modules $B \neq 0$. Recall that a left $R$-module $M$ is faithful if $(0: M)$ $=\{x \in R \mid x M=0\}=\{0\}$.

Theorem 3. Let $R$ be a finite-dimensional torsion-free ring and let $M \neq 0$ be a torsion-free $R$-module. Then $\operatorname{Hom}_{R}(B, M) \neq 0$ for all torsionfree $R$-modules $B$ if and only if $M$ contains a nonzero faithful injective submodule.

Proof. Assume first that $\operatorname{Hom}_{R}(B, M) \neq 0$ for all torsion-free $R$-modules $B \neq 0$. Since $R$ is torsion-free, $E(R)$ is torsion-free and so $\operatorname{Hom}_{R}(E(R), M) \neq 0$. Thus $M$ contains a nonzero injective 
submodule by Lemma 1 . Hence the maximal injective submodule $D$ of $M$, which exists by Theorem 1 , is nonzero. Let $K$ $=\bigcap\left\{\operatorname{Ker} \alpha \mid \alpha \in \operatorname{Hom}_{R}(E(R), D)\right\}$. Then $E(R) / K$ can be embedded in a direct product of copies of $D$. By Lemma $2, E(R) / K$ is a torsionfree $R$-module. Thus $K$ can have no essential extension in $E(R)$ and so $K$ is injective. For any $\alpha \in \operatorname{Hom}_{R}(K, M), \alpha(K)$ is injective by Lemma 1 , and so $\alpha(K) \subseteq D$. Thus $\alpha$ extends to $\beta \in \operatorname{Hom}_{R}(E(R), D)$ and $K \subseteq \operatorname{Ker} \beta$. Hence $\alpha(K)=0$ and so $\operatorname{Hom}_{R}(K, M)=0$. It follows that $K=0$ and thus $E(R)$ is contained in a direct product of copies of $D$. Since any element in $(0: D)$ annihilates any direct product of copies of $D$ and so also $R \subseteq E(R)$, we conclude that $(0: D)=\{0\}$ and hence $D$ is a faithful $R$-module.

Conversely, assume $M$ contains a nonzero faithful injective submodule $D$. If $A \neq 0$ is a left ideal of $R$ then $A m \neq 0$ for some $m \in D$, since $D$ is faithful, and so $\theta: A \rightarrow D$ defined by $\theta(x)=x m$ for all $x \in A$ yields a nonzero member of $\operatorname{Hom}_{R}(A, D)$. Now if $B \neq 0$ is a torsionfree $R$-module then, since $Z(R)=0, \operatorname{Hom}_{R}(B, E(R)) \neq 0$, and so if $0 \neq \alpha \in \operatorname{Hom}_{R}(B, E(R))$ then $A=\alpha(B) \cap R \neq 0$. Then by injectivity of $D$, any nonzero map in Hom $(A, D)$ extends to a nonzero map in $\operatorname{Hom}_{R}(\alpha(B), D)$ and this implies $\operatorname{Hom}_{R}(B, M) \neq 0$, completing the proof.

An interesting consequence of our previous results is the following

THEOREM 4. Let $R$ be a semiprime finite-dimensional torsion-free ring. Then $E(R)$ is a projective $R$-module if and only if $R$ is a semisimple Artinian ring.

Proof. Let $Q$ be a torsion-free injective $R$-module and $F$ a free $R$-module mapping onto $Q$, say $\alpha: F \rightarrow Q$. Now $F \cong \oplus \sum_{\lambda \in \Lambda} R_{\lambda}$ where each $R_{\lambda}=R$ and there is a natural embedding of $F$ in $\oplus \sum_{\lambda \in \Lambda} E\left(R_{\lambda}\right)$ $=M$. Thus $\alpha$ extends to $\beta \in \operatorname{Hom}_{R}(M, Q)$. Since $E(R)=E\left(R_{\lambda}\right)$ is projective, $M$ is a projective $R$-module. By Teply's result [9, Theorem 2.1 ], $R$ has property $\left(^{*}\right)$ and so $M$ is also an injective $R$-module. Thus by Lemma $1, Q$ is a direct summand of $M$ and so $Q$ is a projective $R$-module. Thus every torsion-free injective $R$-module is a torsionless $R$-module in the sense of Bass [2], and since submodules of torsionless $R$-modules are torsionless [2], every torsion-free $R$ module is a torsionless $R$-module. But this is equivalent to $\operatorname{Hom}_{R}(B, R)$ $\neq 0$ for all torsion-free $R$-modules $B \neq 0$. By Theorem $3, R$ contains a faithful injective submodule and so the maximal injective submodule $K$ of $R$ is nonzero and faithful. From Theorem $2, R=K \oplus S$ with $K$ semisimple Artinian. Since $(S K)^{2}=0$ and $S K \subseteq K$ we have $S K=0$ and so $S=0$ since $K$ is faithful. This completes the proof since the converse is trivial. 
The condition that $R$ be semiprime cannot be omitted. For if $R$ is a left Artinian hereditary $Q F-3$ ring (i.e. $E(R)$ is projective) then $R$ is finite-dimensional and $Z(R)=0$ by $[4$, p. 426]. However, $R$ need not be semisimple Artinian.

While prime rings are not necessarily finite-dimensional, a result similar to Theorem 3 holds for torsion-free prime rings. We first have

Lemma 3. A prime ring $R$ is torsion-free if and only if $R$ has a nonzero torsion-free $R$-module.

Proof. If $Z(R)=0$ then $R$ is a torsion-free $R$-module. For the converse, we note that if $K \neq 0$ is an ideal of $R$ then for any left ideal $A \neq 0,0 \neq K A \subseteq K \cap A$, so $K$ is an essential left ideal of $R$. Thus if $Z(R) \neq 0$ then $Z(R / Z(R))=R / Z(R)$ and this would imply every nonzero $R$-module has nonzero singular submodule.

THEOREM 5. Let $R$ be a prime ring having a nonzero torsion-free $R$-module $M$. Then $\operatorname{Hom}_{R}(B, M) \neq 0$ for all torsion-free $R$-modules $B \neq 0$ if and only if $M$ contains a nonzero injective submodule.

Proof. By Lemma 3, $Z(R)=0$ hence $E(R)$ is torsion-free. Thus if $M$ contains a nonzero image of any nonzero torsion-free $R$-module, $M$ has a nonzero injective submodule as in Theorem 3. On the other hand let $M$ contain a nonzero injective submodule $D$. For any torsionfree $R$-module $A \neq 0,(0: A)$ is an ideal of $R$, hence, as in Lemma 3 , if $(0: A) \neq 0$ then $(0: A)$ is an essential left ideal of $R$; but then for any $0 \neq a \in A,(0: A) \subseteq(0: a)$ and this contradicts $Z(A)=0$. Hence $D$ is faithful and as in Theorem $3, \operatorname{Hom}_{R}(B, M) \neq 0$ for all torsion-free $B \neq 0$.

Finally, we consider self-injective rings. In [6], Goldie defined for an $R$-module $M$, the sequence of submodules $Z_{i}(M), i \geqq 0$, where $Z_{0}(M)=0$ and for $i \geqq 1, Z_{i}(M)$ is given by $Z_{i}(M) / Z_{i-1}(M)$ $=Z\left(M / Z_{i-1}(M)\right)$. Moreover, he established that $Z_{i}(M)=Z_{2}(M)$ for all $i \geqq 3$. Consequently for any $R$-module $M, Z_{2}(M)$ can have no essential extension in $M$. In particular if $M$ is injective then $M$ $=Z_{2}(M) \oplus N$; this has also been noted in [7].

THEOREM 6. Let $R$ be a self-injective ring. Then every finite-dimensional torsion-free $R$-module $M$ is a completely reducible injective $R$-module.

Proof. Since $M$ is finite-dimensional every nonzero submodule contains a nonzero uniform submodule, so let $S \neq 0$ be a uniform submodule of $M$. If $0 \neq x \in S$ then $0 \neq R x \subseteq S$ is a torsion-free image of the injective $R$-module $R$ and so, by Lemma $1, R x$ is injective. 
Since $S$ is uniform and $R x$ is a direct summand of $S, R x=S$. Thus any nonzero element of $S$ generates $S$ and so $S$ is a simple injective $R$ module. Letting $M=S \oplus M_{1}$, if $M_{1} \neq 0$ we proceed as above and write $M_{1}=S_{1} \oplus M_{2}$ where $S_{1}$ is a simple injective $R$-module. Continuing in this manner and using the finite dimensionality of $M$, we arrive at the desired conclusion.

COROLlaRy 6. If $R$ is a self-injective ring having property $\left({ }^{*}\right)$, then $R=Z_{2}(R) \oplus K$ (ring direct sum), where $K$ is a semisimple Artinian ring. Hence every torsion-free $R$-module is a completely reducible injective R-module.

Proof. By the remarks preceding Theorem $6, R=Z_{2}(R) \oplus K$. Since $R$ satisfies $\left({ }^{*}\right)$ it has no infinite direct sum of torsion-free left ideals. Hence $K$ is finite-dimensional and torsion-free and thus completely reducible. If $x \in Z_{2}(R)$ then $K x$ is a homomorphic image of $K$ and so isomorphic to a direct summand of $K$. Since also $K x \subseteq Z_{2}(R)$ we conclude that $K x=0$. Now $Z_{2}(R)$ is an ideal of $R$ and so $K Z_{2}(R)=Z_{2}(R) K$ $=0$ and hence the decomposition is two-sided. That every torsionfree $R$-module is injective and hence completely reducible now follows from [1, Theorem 3.1].

Any quasi-Frobenius ring is both left Noetherian and self-injective, so that Corollary 6 provides an extension of [7, Proposition 2.8].

ADDED IN PROOF. We have noticed that R. N. Gupta, using different methods, has also obtained Theorem 4, see Osaka J. Math. 5 (1968), Theorem 4.1.

\section{REFERENCES}

1. J. S. Alin and S. E. Dickson, Goldie's torsion theory and its derived functor, Pacific J. Math. 24 (1968), 195-203. MR 37 \#2834.

2. H. Bass, Finitistic dimension and a homological generalization of semi-primary rings, Trans. Amer. Math. Soc. 95 (1960), 466-488. MR 28 \#1212.

3. H. Cartan and S. Eilenberg, Homological algebra, Princeton Univ. Press, Princeton, N. J., 1956. MR 17, 1040.

4. E. Gentile, Singular submodule and injective hull, Nederl. Akad. Wetensch. Proc. Ser. A $65=$ Indag. Math. 24 (1962), 426-433. MR 26 \#2478.

5. A. W. Goldie, Rings with maximum condition, Lecture Notes, Yale University, New Haven, Conn., 1961. \#2282.

6. - Torsion-free modules and rings, J. Algebra 1 (1964), 268-287. MR 29

7. M. Harada, Note on quasi-injective modules, Osaka J. Math. 2 (1965), 351-356. MR 34 \#306.

8. M. Teply, Torsion free injective modules, Pacific J. Math. 28 (1969), 441-453.

9. - Some aspects of Goldie's torsion theory, Pacific J. Math. 29 (1969), $447-460$.

University of TeXas 